which it purports to offer, and that it is better to leave the examiners with discretion to reject candidates who appear not to attain the required standard of proficiency. The recommendation therefore has not been retained. The recommendation that in the three primary subjects no candidate should be allowed to pass who fails to obtain $50 \%$ of the marks assigned to the clinical examination is repeated, but certain other minima suggested in that recommendation have been dropped. They are considered matters proper to be determined by the examining bodies, and indeed the general effect of such alterations as have been made in the conduct of examinations is to leave these bodies with a larger discretion.

\section{Reports of Societies}

\section{MANAGEMENT OF PATIENTS WITH ESSENTIAL HYPERTENSION}

In the Section of Medicine of the Royal Society of Medicine on April 22, Dr. MaUrice Davidson presiding, a discussion took place on the management of patients with essential hypertension.

Dr. Geoffrey Evans, discussing aetiology, said that in some of these cases there was undoubtedly a constitutional disease, to which at least there was an inherited predisposition; in others family influence and environment might determine the development of hypertension. Thus considerable understanding of a patient was necessary before giving him advice as to his manner of living, but at least it should be remembered that living was an art and nowadays something of a problem. In the emotional background of these people there was often fear and apprehension, thus it was helpful to cultivate a sanguine attitude of mind towards them. Exhaustion aggravated hypertension even if it did not cause it ; rest and relief from emotional tension were important. As for sedatives, he criticized the extravagance with which phenobarbitone and the barbiturates were prescribed. Exercise and a little alcohol might do good; those with peripheral congestion might benefit from venesection, and those overweight by weight reduction. Those were the ordinary conservative measures of approach for patients with essential hypertension.

\section{Value of Potassium Thiocyanate}

Potassium thiocyanate, Dr. Geoffrey Evans continued, was of undoubted value in the relief of headache and giddiness, and in some patients improved their feeling of well-being. In the minority there was a fall in blood pressure. He mentioned the case of a scientist, aged 42 , who had hypertensive retinopathy and was given potassium thiocyanate. He had now been relatively well for five years except for a recurrence three years ago, when he gave up the treatment for a time and his headaches immediately returned. He was now, with this treatment, able to do rather more than two-thirds of his day's work. It was strange that after 11 years' experience of potassium thiocyanate there should still be such difference of opinion as to its value. He believed the reason to be as follows. Hypertension was caused by generalized hypertonia in the systemic circulation, and many of its symptoms and complications were brought about by localized vascular spasm. If such spasm caused headaches and functional disturbance the patient might have these symptoms for years and apparently be no worse. But in some patients there followed a structural reaction to the functional change; this took the form of arteriosclerosis with irreversible changes, leading to thrombosis and other sequelae. As physicians they had to determine by investigation whether the arteriosclerdic disease was active or quiescent. Their patients would do well, not necessarily according to the way in which it was possible to control the symptoms-the headaches, giddiness, and so on-but according to the nature of the arteriosclerosis. In some the arteriosclerosis might be quiescent and perhaps disappear ; in others, once started, it was steadily progressive. Patients might respond, not only symptomatically but in their blood pressures, to potassium thiocyanate, and show only moderate hypertension, and yet develop retinal thrombosis because the disease was not controlled. So far as he knew there was no method, other than the conservative measures he had detailed, of preventing the active phase of arteriosclerotic disease developing. It was on this that the prognosis turned, and it could be evaluated only by watching a patient with arteriosclerotic disease treated on conservative lines over a period of ten or twenty years.

\section{Surgical Measures for Relief of Hypertension}

Mr. A. Dickson Wright said that hypertension was one $\mathrm{cf}$ the most inexact and bewildering of diseases. One of its types was secondary to migraine. Sufferers from migraine were four times as likely as the ordinary person to get hypertensio?. Another group consisted of children with headaches and dizziness which would be described as functional if they occurre 1 in the adult. The method of dealing with hypertension by means of surgery lay in one of two directions. One method was to attack the adrenals, but no very large measure of success had been achieved. The other method was to attack through the sympathetic system. Mr. Dickson Wright showed by means of a film the procedure he favoured, which was Adson's operation of sympathectomy and partial adrenalectomy. This operation should not be undertaken if the patient was over 50 or had a high blood pressure which did not subside after rest in bed and sedation. Of bad significance were high diastolic pressure and high pulse pressure. The pale hypertensive had a much worse prognosis than the bucolic subject. Women did better than men in every respect. But if a case was around the forties, and there were headaches and other symptoms of hypertension, one should not be too strict in looking for contraindications. While his own predilection was for the Adson operation, there were others which were employed by other surgeons, and at the Mayo Clinic he was informed that the results were considered to be slightly better with the Smithwick operation. While in his hands Adson's operation had seemed to have a definite value, he never trusted himself to compile statistics of results. There was a certain percentage of failures, but no one was the worse for this operation and it had practically no complications.

Dr. C. Wyndham described some recent work carried out at the British Postgraduate Medical School on peripheral mechanisms which affect blood pressure. These mechanisms were skin tone, muscle tone, and splanchnic tone, which affected not only total peripheral resistance but also venous tone, and this in its turn affected the pressure with which the blood returned to the right heart and thus affected the cardiac output. He described in this relation the action of sympathectomy.

Mr. H. J. B. AtKINS asked whether there might not be some danger in the sudden reduction of blood pressure after sympathectomy. One of Mr. Dickson Wright's two fatalities in his large series of cases was due to cerebral thrombosis, and in a very small series of the speaker's a patient had a retinal thrombosis on the fourth day and went blind in one eye. If this complication of post-operative thrombosis was a serious danger he wondered whether it might not be wise to institute routine heparinization.

\section{A "Forty-hour Week" for the Heart}

Dr. Evan BeDFord said that by far the most important cause of death in hypertension was heart failure. Over $60 \%$ of patients with persistent hypertension had heart failure of one form or another, compared with less than $20 \%$ dying of cerebral vascular accidents. Therefore the management of the patient should be directed especially to the care of the heart. Probably half of all cases of organic heart disease seen in practice were due wholly or in part to essential hypertension. If the rate at which the heart enlarged were measured it would be found to be almost imperceptible from year to year. In cases in which he had made measurements over a period up to 14 years the average increase in transverse diameter was less than $2 \mathrm{~mm}$. annually, but at a later stage a rapid enlargement might take place. Coronary disease must always be remembered as a frequent complication of hypertension. A fair number of cases had combined coronary and hypertensive heart disease. Treatment should be directed towards preventing or delaying the enlargement of the heart and, when the heart was enlarged, towards preventing or delaying heart failure. The crux of treatment was the control of the hypertension itself, but as yet no sure way of control was known. No drug was capable of permanently lowering the blood pressure in a case of essential 
hypertension. Occasional successes were obtained with thiocyanate, but in the majority of cases thiocyanate was either too toxic or too disappointing to be any solution of their problems as a whole. The hypertensive patient must be taught to live slowly: a "forty-hour week" should be prescribed for the heart. By judicious advice much could be done to delay the progress of cardiac enlargement and the onset of heart failure. As for surgery, it was most difficult to assess the results from the statistics. It was unfortunate that results were given in terms of reduction of diastolic pressure, which meant very little. In terms of the humoral theory of hypertension surgery was an irrational procedure, but it might still be an effective one. It was not a cure for hypertension, but at the best it could lower, sometimes appreciably, the blood pressure level and diminish the burden on the heart.

Mr. Dickson WRIGHT said that the incidence of clotting in the eyes or brain after these operations of sympathectomy had been low, and rather comparable with the incidence of femoral thrombosis after abdominal operations. He had not yet thought of giving heparin. It was the load on the heart which killed the patient. They were too prone to think of cerebral haemorrhage, but the real mischief was the slow wearing out of the myocardium.

\section{MEDICAL PHOTOGRAPHY}

A meeting of the Medical Society of the L.C.C. Medical Service was held at County Hall on April 3 to discuss the uses of photography in medicine.

Dr. J. E. MCCARTNEY stressed the value of accurate and immediate recording of medical cases. Lesions were often fleeting, appearances changed rapidly, patients might recover or die, and information not recorded at the time might be impossible to get later. In a written record the clinician put down only what he saw. A sketch might add a few more details, but a photograph would reveal not only all the clinician saw at the time but perhaps further information for other observers. Accurate photographic records were valuable both for present and for future investigations. Medical photography should be taken seriously. It required adequate modern equipment and a skilled staff. Medical photography was so specialized and involved such varying conditions of work that it was imperative to employ a first-class, well-trained, well-paid photographic technician.

Each patient presented a different problem, and special pieces of apparatus and a number of different lenses and cameras were necessary to cover the whole range of clinical photography. Special equipment was again required for biopsy or post-mortem specimens. To show the correct dimensions of the specimen a centimetre scale or similar measure should be placed beside it. Miniature photography was useful for recording case-papers, documents, reprints, books, etc. This had been specially developed in America with elaborate and costly apparatus.

Dr. McCartney went on to say that in America, during his recent visit, he had seen the latest developments. of colour work and printing. Illustrations demonstrating the value of coloured backgrounds for throwing the subject into relief were shown. Cinephotography was invaluable for recording movement, such as gaits and the progress of orthopaedic and similar cases. The use of colour in the cinephotography of operations was demonstrated, and the value of sound film for teaching purposes was stressed. A $16-\mathrm{mm}$. film showing parts of various medical films, in black and white and in colour, made by Mr. J. E. Andrews, the L.C.C.'s medical photographer, was then demonstrated.

Dr. GuNN emphasized the value of cinephotography for teaching students, but thought it should only be considered in the case of operations or manipulations, as an adjunct to direct clinical teaching. Mr. MATHESON remarked that though colour photography had considerably improved films of operations, it was still very difficult to see important details, owing to the shiny appearance due to reflection of light from the tissues.

Dr. MCKerTH, raising the question of film-strips, suggested that for teaching purposes film-strips were more useful than actual moving films as they could be arranged beforehand to show all the points, and only those points, which the lecturer desired to stress. He also questioned the value of taking films of operations. It was impossible to learn to operate from the film, and, while it might make a pretty picture, there were many other aspects of medicine in which a film could be of much greater value.

Mr. PIERCey, admitting that part of the film shown was of himself doing a thyroidectomy, said that the reason for having this film taken was to preserve a record of Mr. C. A. Joll's technique and particularly of his exposure and ligature of the inferior thyroid artery. He thought that films of operations showing special technique were of great interest to surgeons and others. Mr. ANDrews said it was quite easy to get filmstrips made by commercial firms. The cost was about $1 \mathrm{~s}$. a picture and projectors were available from about $£ 8$ upwards. He thought that slides were more generally useful. The order of showing them could be altered and they stood up to heat much better than film-strips.

\section{Correspondence}

\section{State Medical Service in New Zealand}

SIR,-New Zealand has enjoyed a partial State Medical Service for the past 10 years. In the current issue of St. Mary's Hospital Gazette Mr. A. E. Porritt-a New Zealander coming to England as a Rhodes Scholar to study medicine 23 years ago-gives a "dispassionate review on things medical in New Zealand" which he observed during a recent visit to that country. He remarks that although New Zealand is one of the most beautiful and naturally endowed countries in the world to-day "it is even more full of petty restrictions in its administration than this country." He sums up his conclusion on the medical services in these pregnant sentences: (a) A slightly improved all-round service for the public-during business hours. To get a doctor in New Zealand after 6 p.m. and over week-ends is not an enviable task. (b) A definite falling off in the standard of medical practice from both ethical and clinical viewpoints. This is a consensus of opinion from all branches of the profession. (c) A growing and already appreciable dearth of specialists-and this in a country where the proportion of men taking higher degrees was previously exceptionally high.

We have been warned !-I am, etc.,

London, S.W.1.

\section{E. Graham-Little.}

\section{Reiter's Syndrome}

SIR,-I am particularly interested in the claim made by $\mathrm{Dr}$ R. R. Willcox and his collaborators (April 12, p. 483) that gold salts (myocrisin) were curative in two of their cases of Reiter's disease. As reported in an address to the Medical Society for the Study of Venereal Diseases in May, 1944, I was at that time carrying out treatment with myocrisin on a typical case of Reiter's disease with non-gonococcal urethritis, bilateral conjunctivitis, polyarthritis, and keratodermia blennorrhagica (Case 20 in the series) in spite of the fact that no pleuropneumonialike organisms had been cultivated from the urethral, conjunctival, or skin lesions. I had read the paper of Findlay et al. in which it was stated that organic gold salts protected rats and mice from developing arthritis after injection in the pad with pleuropneumonia-like organisms, and the drug was prescribed on the supposition that the inclusions seen in my cases may have been the granular phase in the development of these organisms. No beneficial effects followed gold therapy in this and two further cases under my care. King in the treatment of Reiter's disease with the Simpson-Kettering hypertherm gave several cases injections of myocrisin during fever and the results obtained were no better than with fever alone. I was interested to note that in Case 1 of the authors' there was a fall in the blood sedimentation rate during treatment, as in none of my cases has this ever been observed ; in fact I find the test of little value in the assessment of progress. In one apyrexial case now under my care that has already received five injections of myocrisin the B.S.R., which was originally 30 , has risen steadily each week and is now 41 .

It is possible that the beneficial effects of gold therapy in the cases recorded by Willcox et al. were non-specific and due to the rise in temperature which sometimes occurs after the 